\title{
Self-designation and group allocation
}

\author{
John Craven ${ }^{1}$
}

Accepted: 4 February 2022 / Published online: 4 March 2022

(C) The Author(s) 2022

\begin{abstract}
A widely held rights-based view asserts that individuals should be permitted to selfdesignate characteristics such as race and gender. But some argue that there are opinions that oppose the use only of self-designation, and that these should not be ignored. Kasher and Rubinstein (Logique Anal 160:385-395, 1997) demonstrated that the latter view is equivalent to accepting that one or more of five conditions must be violated. This paper extends their analysis to allow for more than two categories, and focuses on simplifying the conditions without jeopardising their conclusion. In particular, we use significantly weaker conditions of independence and of symmetry than are common in other extensions of the original proposition.
\end{abstract}

Keywords Collective decision-making $\cdot$ Social choice $\cdot$ Liberalism

\section{Introduction}

There is significant controversy in public debate about the right of people to be included in certain categories of, for example, gender, religion, race or sexual orientation, only on the basis of their own self-designation. For practical purposes, inclusion can allow or restrict access to particular facilities, such as religious premises and gender-specific changing rooms, or affect participation in sports and other activities. There are many people who believe that an individual has the right to decide their category without intervention from others, and that non-discrimination principles then influence the consequences. Others disagree, arguing that not all self-designations should be allowed to pass unchallenged. In some circumstances, such as national censuses or large social surveys, the only information collected is individual self-designations. Then critics do not accept conclusions based on that information base, so that, for example, they do not agree that a

John Craven

cravenjag17649@gmail.com

1 Department of Philosophy, King's College, London WC2R 2LS, UK 
national census gives a true picture of the percentage of the population who are 'really' in some category.

Kasher and Rubinstein (1997) establish a social choice framework to analyse this topic. They formalise a context which arises from the Israeli 1950 'Law of Return' that states criteria for eligibility as a citizen (see for example, Jewish Agency 2019). These criteria are more extensive than the definition of Jewishness under Halachic law, and the resulting immigration has led to political controversy around, for example, the observance of the Sabbath (Reeves 2011; Galili 2020).

Following Kasher (1993), K\&R (page 386) present the problem as follows:

...each of $n$ individuals in a society holds a view with respect to every individual, including oneself, whether the latter is a J. The collective identity of $\mathbf{J}$ is determined by the individual views of "who is a $\mathrm{J}$ ". The method of determining who is a $\mathbf{J}$ is viewed as a function which assigns a meaning to "who is a $\mathrm{J}$ " for each profile of all the individual views.

In other words, the function takes account of both self- and other-designation.

$\mathrm{K} \& \mathrm{R}$ base their analysis on this important and controversial context, and their principal result (theorem 1(a) page 389) is to show that five conditions (axioms in $\mathrm{K} \& \mathrm{R})$ together entail that self-designation is the only method of determining group membership. So a critic who wants to deny that it is always appropriate to use selfdesignations in this way must reject one of more of these conditions. This paper develops this topic by considering designations into more than two categories, and presents alternative versions of the conditions that suffice to show the principal result. Social choice problems conventionally involve the aggregation of individual opinions concerning set of objects. In the tradition of Arrow (1951) and Sen (2017), the opinions are expressed as rankings of the set of objects. In the tradition of Wilson (1975) and Rubinstein and Fishburn (1986), the opinions are expressed as classifications or partitions of the set of objects. The unconventional aspect of K\&R is that the set of objects is identical to the set of individuals. Then the opinions are expressed as a set of self- and other designations of each individual by each individual. Although most of the conditions introduced by $K \& R$ are related to conditions in conventional social choice, they must be adapted to allow for the identity of the objects and the individuals. These adaptations change the focus from conditions that necessarily entail that the outcome is determined by a dictator to conditions that necessarily entail that the outcome is determined by self-designation alone.

This unconventional extension of social choice does not lose the typical assumption that the only information that is relevant to the determination of the outcome of the social choice process are the individual opinions. If a critic of Israel's 'Law of Return' wishes to assert that an individual can be considered to be a $\mathbf{J}$ just if their mother is a $\mathbf{J}$, the information base used to determine 'who is a $\mathbf{J}$ ' extends beyond (and maybe excludes) the self- and other-designations invoked by $\mathrm{K} \& \mathrm{R}$. Of course, any of the individuals in the society might base their self- and other-designations on that maternal principle, but the social choice approach allows that this opinion might not prevail, given the opinions of others. 
Although our concern is with the basic ethical question of the consequences of rejecting liberalism - that is rejecting self-designation as the basis for devising categories - a similar framework can be used in decision-making contexts. For example, a musicians' convention might wish to divide its delegates into groups such as jazz-musicians and blues-musicians, and decide to do so on the basis of each delegate's designation of herself and of all the other delegates. If the convention adopts the five conditions presented here, then the outcome must be that every delegate is located where she self-designates. In common with conventional social choice, the methods can be used to make practical decisions and devise social outcomes as well as to offer a critique of principles that might be held by a single individual.

An alternative decision-making context might reject the possibility that the outcome is determined by self-designation. For example, ${ }^{1}$ students engage in a group project and each student evaluates her own and the other students' contributions as 'fail', 'pass', 'merit' or 'distinction'. The tutor aggregates these evaluations into grades, and it is possible that the grade for one student depends in part on her own self-evaluation, but tempered by the views of others. It might seem unacceptable that there are any circumstances in which a student's grade depends only on her self-evaluation given the temptation to overstate her own contribution. In that context, one or more of the five conditions must be rejected to avoid an unacceptable outcome. The same argument would apply in the two-category case of $\mathrm{K} \& \mathrm{R}$, where the grades available are 'pass' and 'fail'.

The extension of the framework introduced by $K \& R$ to multiple categories has been examined by, for example, Houy (2007) and Cho and Ju (2017). Some of the conditions that they use are different from those considered here, and are arguably less closely related to the five conditions in K\&R. Miller (2008) also considers multiple categories and the allocation of individuals when categories are subdivided or combined. Then we could compare the membership of the jazz group at the musicians' convention with the membership of the trad-jazz and modern-jazz groups if such a subdivision were introduced or abolished.

Much of the literature following K\&R has been concerned with decision-making issues. For example, Samet and Schmeidler (2003) consider the extent of consent from others that is needed to allow an individual to self-designate, Alcantud and Laruelle (2020) consider the formation of 'clubs' using voting rules and involving vetoes, and Houy (2007) and Fioravanti and Tohmé (2020) constrain the influence of individuals so that, for example, existing members of a category have the privileged ability to admit others to that category. These are not our question, which remains the examination of the choice between accepting that the outcome results only from self-designation and rejecting one or more of the conditions.

\footnotetext{
${ }_{1}$ I am grateful to an anonymous referee for this example.
} 


\section{Formal specification}

There is a finite set $I$ of $n$ individuals. Each individual designates each individual into one of $2 \leq r<n$ categories $C_{1} \ldots C_{r}$. A profile $\Pi$ is an $n \times n$ matrix in which $\Pi_{i j}=C_{s} \equiv$ "individual $i$ designates individual $j$ in $C_{s}$ ". The $\Pi_{i i}$ are the selfdesignations. The rows of $\Pi$ are the designations made by each individual; the columns of $\Pi$ are the designations of each individual. For any $\Pi$, an aggregator $f$ determines an outcome $q=f(\Pi)$ which is an $n$-vector that locates each individual in exactly one of the categories. So $q_{i}=C_{s} \equiv$ " $i$ is located in $C_{s}$ in the outcome". The domain of the aggregator is unrestricted: any individual can designate any individual (including herself) in any category, and the aggregator must then determine an outcome.

The liberal aggregator $f^{L I B}$ determines that, for all $\Pi$ and for all $i \in I, q_{i}=f_{i}^{L I B}(\Pi)=\Pi_{i i}$. In line with $\mathrm{K} \& \mathrm{R}$, but in the more general circumstances, theorem 1 in section 3 shows that $f^{L I B}$ is the only aggregator that satisfies the five conditions that are presented in this section.

\subsection{Unanimity}

This is a straightforward condition on $f$ that echoes those used in conventional social choice theory. It ensures that the outcome reflects all unanimous designations and non-designations:

Unanimity: $\forall j \& \forall s: \forall i \Pi_{i j}=C_{s} \rightarrow q_{j}=C_{s}$ and $\forall i \Pi_{i j} \neq C_{s} \rightarrow q_{j} \neq C_{s}$.

$\mathrm{K} \& \mathrm{R}$ label this condition 'consensus'.

\subsection{Weak independence}

Most explorations in conventional social choice theory involve some unanimity (or Pareto) condition along with an independence condition. The most famous of the latter is Arrow's (1951) 'independence of irrelevant alternatives' that specifies that the social choice (or ranking) between two alternatives depends only on the individuals' rankings of those two alternatives. In some investigations of decisionmaking, this condition is replaced by a condition of non-manipulability (Gibbard 1973; Satterthwaite 1975), reflecting the fact that strategic voting, for example, is effectively ruled out if decisions on pairs of alternatives are made independently of stated views about other alternatives.

A strong form of independence appears in examinations of self- and otherdesignation into categories. For example, Cho and Ju (2017) use 'independence of irrelevant opinions' which specifies that the outcome location of any individual depends only on the designations of that individual. That is significantly stronger than the condition used by $K \& R$. In the multi-category case, the condition that we use is that a change of one or more designations can lead to a relocation of $j$ only if either some individual re-designates $j$, or if the change also leads to other relocations. If no-one re-designates $j$, the impact of other changes cannot be only on $j$. K\&R label their condition independence (I), in contrast to the more stringent $\mathrm{I}^{*}$ 
which they use in a different result, and which is similar to the condition used by Cho and Ju (2017). To emphasise the lesser applicability of the condition used here, we label it:

Weak independence: $\quad \forall j \& \forall \Pi, \Pi^{\prime}\left[\left[\forall i \Pi_{i j}=\Pi_{i j}^{\prime} \& \forall k \neq j q_{k}=q_{k}^{\prime}\right] \rightarrow q_{j}=q_{j}^{\prime}\right]$ where $q=f(\Pi) \& q^{\prime}=f\left(\Pi^{\prime}\right)$.

This condition has analogies in other interconnected contexts. For example, if the government imposes a tax on tea and coffee, then a partial equilibrium analysis of the coffee market alone asserts that the price of coffee (as paid by the coffeedrinker) rises. A general market analysis indicates that people will switch to other drinks so that the price of cocoa rises, and also that the price of tea kettles is likely to fall. Further income effects on many other markets might follow because people who drink a lot of coffee and tea have less to spend on other things. So, the price of a good can change either because of direct effects of interventions in its own market, or because of indirect effects from interventions in other markets. What is extremely unlikely (here excluded by weak independence) is that the only impact of the tax on coffee is a change in the price of cocoa to which no tax has been applied.

Weak independence allows these sorts of 'system effects'. For example, suppose that $i$ re-designates $j$ from $C_{s}$ to $C_{t}$ and this leads to a change in the outcome location of $j$. Many other individuals might feel strongly that $k$ should be colocated with $j$ because of their similarities, and an aggregator that reflects this widespread view of colocation would also change the outcome location of $k$, even though no individual has re-designated $k$. The stronger independence condition would not allow this secondary effect.

\subsection{Weak monotonicity}

In conventional social choice, independence conditions are often accompanied by monotonicity (or positive association) conditions, for the simple reason that the former specify that additional support for some outcome can change that outcome, but do not rule out the possibility that the change is perverse. Monotonicity conditions ensure that additional support reinforces an outcome decision.

Here, a simple monotonicity condition would ensure that if $i$ re-locates $j$ from $C_{t}$ to $C_{s}$, and before the change $q_{j}=C_{s}$ then $j$ is not re-located out of $C_{s}$ as a result of the change. However, the relocation of $j$ by $i$ might lead to system effects that potentially complicate the analysis. It is sufficient for our purposes that the monotonicity condition is applicable only when each individual other than $j$ is unanimously designated and so system effects are excluded by unanimity.

$$
\begin{aligned}
& \text { Weak monotonicity: } \forall j \& \forall s \& \forall \Pi, \Pi^{\prime}:\left[\left(\Pi_{i j} \neq C_{s} \& \Pi_{i j}^{\prime}=C_{s}\right) \&(\forall k \neq\right. \\
& \left.\left.i \Pi_{k j}^{\prime}=\Pi_{k j}\right) \&\left(\forall i, \forall k \neq j \Pi_{i k}=\Pi_{k k}=\Pi_{i k}^{\prime}\right) \& q_{j}=C_{s}\right] \rightarrow q_{j}^{\prime}=C_{s} \quad \text { where } \\
& q=f(\Pi) \& q^{\prime}=f\left(\Pi^{\prime}\right) .
\end{aligned}
$$

This is a less demanding condition than the monotonicity condition used by $\mathrm{K} \& \mathrm{R}$ because we have limited its applicability to profiles in which each individual other than $j$ is unanimously designated. A stronger monotonicity condition would prevent the relocation of $j$ out of $C_{s}$ when $i$ re-locates $j$ from $C_{t}$ to $C_{s}$ even if system effects 
occurred. Using the stronger condition would leave open the possibility of arguing that the system effects had themselves weakened the case for locating $j$ in $C_{s}$-and as our theorem does not need a stronger condition we can avoid the possibility of such arguments using weak monotonicity.

\subsection{Non-discrimination}

In conventional social choice, an anonymity or symmetry condition ensures that, if two profiles differ only because two individuals' opinions about the objects are swapped, then the outcomes from the profiles are the same. It is also used to require that there is no discrimination in the outcome for a profile when each individual has the same opinion about two objects: the 'rules' for both objects are the same. Symmetry of either form has an obvious 'democratic' appeal.

In our context, a symmetry condition would require identity of outcomes for $i \& j$ if their positions are swapped both in their role as designators, and in their role as designatees. Cho and $\mathrm{Ju}$ (2017) have asserted that 'name changes shift no fundamental content' (page 519) and Samet and Schmeidler (2003) that 'a rule does not depend on the names of individuals' (page 216) and invoke symmetry conditions that have relatively wide applicability, thereby, incidentally, excluding common tie-breaking mechanisms. A symmetry condition that generalises that used in $\mathrm{K} \& \mathrm{R}$ would be:

Symmetry: $\forall \Pi \& \forall i, j\left[\left[\Pi_{i i}=\Pi_{j j} \& \Pi_{i j}=\Pi_{j i} \& \forall k \neq i, j\left(\Pi_{k i}=\Pi_{k j} \& \Pi_{i k}=\right.\right.\right.$ $\left.\left.\left.\Pi_{j k}\right)\right] \rightarrow q_{i}=q_{j}\right]$ where $q=f(\Pi)$.

This condition requires that $i \& j$ are designated similarly by all other individuals and that all other individuals are designated similarly by $i \& j$.

We use a different condition in the proof of theorem 1 by limiting the similarity of designation to a restricted set of circumstances. We say that $i$ is an $s \backslash t$ singleton just if $i$ self-designates in $C_{s}$, and every other individual designates $i$ in $C_{t}$. The proof of theorem 1 requires that the aggregator does not discriminate between $s \backslash t$ singletons because all are co-located in the outcome:

Non-discrimination: $\quad \forall \Pi \& \forall s, t \& \forall i, j\left[\left(\Pi_{i i}=\Pi_{j j}=C_{s}\right) \& \forall k \neq i \Pi_{k i}=\right.$ $\left.\left.C_{t}\right) \&\left(\forall k \neq j \Pi_{k j}=C_{t}\right)\right] \rightarrow q_{i}=q_{j}$ where $q=f(\Pi)$.

This differs from symmetry in two ways. First, non-discrimination requires that $\forall k \neq i, j \Pi_{k i}=\Pi_{k j}=\Pi_{i j}=\Pi_{j i}$. Second, non-discrimination places no restriction on the $\Pi_{i k}$ and $\Pi_{j k}$ for $k \neq i, j$.

Section 4 explores the difference between the implications of symmetry and nondiscrimination.

\subsection{Accountability}

The final condition in $K \& R$ does not have a parallel in conventional social choice theory because it refers to the possibility of privileged status for self-designation over other designation. This privilege clearly applies if the aggregator is $f^{L I B}$, for 
which a category is empty in the outcome just if no individual self-designates in that category. Any other aggregator prevents some individual from being located where she self-designates in some profile presumably because, in some sense, sufficient others designate her elsewhere.

The 'Liberal Principle' that is introduced by K\&R requires that if $i$ selfdesignates in $C_{s}$, then $i$ can be located elsewhere in the outcome only if some other individual is located in $C_{s}$. The condition allows the following accountability narrative:

$i$ self-designates in $C_{s}$, but is located in $C_{t}$ in the outcome. $i$ questions why this is so, and the reason is given that some other individual $j$ has a greater claim to be located in $C_{s}$, and that $i$ and $j$ are sufficiently distinct that they should not be co-located.

For example, if $i$ is an $s \backslash t$-singleton and every individual other than $i$ designates $j$ in $C_{s}$, no-one designates $i \& j$ in the same class. So no-one regards $i \& j$ as similar enough that they should be located together. If the weight of opinion leads to the location of $j$ in $C_{s}$, it also supports the conclusion that $i \& j$ are sufficiently different that they should not be co-located. So $i$ would not be located in $C_{s}$ despite her selfdesignation there.

Formally, the condition is:

Accountability: $\forall \Pi \& \forall j \& \forall s\left(\Pi_{j j}=C_{s} \& q_{j} \neq C_{s}\right) \rightarrow \exists k \neq j q_{k}=C_{s}$ where $q=f(\Pi)$.

Arguably, the condition is better called accountability because the more common use of the term 'liberal' in conventional social choice (such as Sen 1970) describes a process in which it is given that one decision depends only on the opinion of a single individual who has some special status or interest in relation to that decision.

In conjunction with unanimity, accountability allows that there are profiles in which $i$ 's outcome category is the same as her self-designation even though no other individual designates $i$ in that category. If every individual other than $i$ is unanimously designated other than in $C_{s}$, and only $i$ designates $i$ in $C_{s}$, then the two conditions entail that $i$ is located in $C_{s}$. Accountability precludes the general imposition of a requirement that, if $i$ self-designates in $C_{s}$, then $i$ can only be located in $C_{s}$ if at least one other individual designates that $i$ is in $C_{s}$. So it is this condition and the associated accountability narrative that might be felt to be unreasonable in the student assessment example given in the introduction. But in other contexts, including the census categorisation and the allocation of convention delegates to sub-groups, the accountability condition and associated narrative are arguably more acceptable.

\section{The theorem}

The main result of this paper is 
Theorem 1 If the domain of the aggregator is unrestricted, $f^{L I B}$ is the only aggregator that satisfies unanimity, weak independence, weak monotonicity, nondiscrimination and accountability.

As noted above, accountability and unanimity together allow that there are specific profiles for which any individual's self-designation determines her outcome despite opposition from all other individuals. The theorem entails that, under the five conditions, this determination must occur for all profiles.

The proof follows a similar strategy to the proof of theorem 1(a) in K\&R. It requires the use of four profiles $\Pi^{1} \ldots \Pi^{4}$ for which the outcomes using some aggregator $f$ are $q^{1} \ldots q^{4}$. In each case, unanimity ensures that the outcome location of any individual is one of the categories into which that individual is designated by herself or another.

Profile If the theorem is not true, we can identify $\Pi^{1}$, some $h$ and some $s \& t$ $\Pi^{1}$ : $\quad$ such that $\Pi_{h h}^{1}=C_{s} \& q_{h}^{1}=C_{t}, t \neq s . h$ self-designates in $C_{s}$ but is not located there in $q^{1}$ and so $f \neq f^{L I B}$.

Profile $\quad \forall i \Pi_{i h}^{2}=\Pi_{i h}^{1} \& \forall j, j \neq h \forall i \Pi_{i j}^{2}=q_{j}^{1}$.

$\Pi^{2} \quad$ Every individual designation of $h$ is the same as in profile $\Pi^{1}$. Every individual designation of each other individual $j$ is the same as the location of $j$ in the outcome for profile $\Pi^{1}$. Unanimity entails $\forall j \neq h q_{j}^{2}=q_{j}^{1}$. In addition, no individual designation of $h$ has changed from $\Pi^{1}$, and weak independence entails that $q_{h}^{2}=q_{h}^{1}=C_{t}$.

Profile $\quad \Pi_{h h}^{3}=\Pi_{h h}^{2}=C_{s} \& \forall i \neq h \Pi_{i h}^{3}=C_{t}=q_{h}^{2} \& \forall i, \forall j \neq h \Pi_{i j}^{3}=\Pi_{i j}^{2}$.

$\Pi^{3}$ : $\quad$ Every individual designation is the same as in $\Pi^{2}$ except that any individual who did not other-designate $h$ in $C_{t}$ in $\Pi^{2}$ designates $h$ in $C_{t}$ in $\Pi^{3} . h$ continues to self-designate in $C_{s}$, and so $h$ is an $s \backslash t$-singleton in $\Pi^{3}$.

All individuals except $h$ are unanimously designated in $\Pi^{3}$, and, compared with $\Pi^{2}$ additional individuals designate $h$ in $C_{t}$. Weak monotonicity entails that $q_{h}^{3}=q_{h}^{2}=C_{t}$ and accountability entails that $\exists j \neq h q_{j}^{3}=C_{s}$ because $\Pi_{h h}^{3}=C_{s}$ but $q_{h}^{3} \neq C_{s}$. So $K=\left\{i \mid q_{i}^{3}=C_{s}\right\} \subset I$ is non-empty and $h \notin K$. $K$ consists of the individuals who are located in $C_{s}$ in $q^{3}$. For convenience, $K^{\prime}=K \cup\{h\}$.

Profile $\quad \forall i \Pi_{i h}^{4}=\Pi_{i h}^{3} \& \forall j \in K\left(\Pi_{j j}^{4}=C_{s} \& \forall i \Pi_{i j}^{4}=C_{t}\right) \& \forall i \forall j \notin K^{\prime} \Pi_{i j}^{4}=\Pi_{i j}^{3}$

$\Pi^{4}$ : All members of $K^{\prime}$ are $s \backslash t$-singletons, and by the definition of $K^{\prime}$, every $j \notin K^{\prime}$ is unanimously designated other than in $C_{s}$. Unanimity entails $\forall j \notin K^{\prime} q_{j}^{4} \neq C_{s}$ so that non-discrimination and accountability together entail that all members of $K^{\prime}$ are located in $C_{s}$ in $q^{4}$. In particular, $h \in K^{\prime}$ so that $q_{h}^{4}=C_{s} \neq q_{h}^{3}=C_{t}$.

For all $j \in K q_{j}^{3}=q_{j}^{4}=C_{s}$ and for all $j \notin K^{\prime} q_{j}^{3}=q_{j}^{4} \neq C_{s}$ so that for all $j \neq h q_{j}^{3}=q_{j}^{4}$, and no individual (including $h$ ) changes her designation of $h$ between $\Pi^{3} \& \Pi^{4}$. So $q_{h}^{4} \neq q_{h}^{3}$ violates weak independence. 
Starting with the supposition in $\Pi^{1}$ that the theorem is not true, we have reached a conclusion about $q^{3} \& q^{4}$ that does not satisfy all the conditions. So the theorem is true.

\section{Preponderance aggregators}

In some contexts, such as the project-grading example, accountability might not be acceptable. When there are only two categories (pass and fail), the outcome for each individual can be determined by, for example, a majority aggregator when $n$ is odd. This aggregator satisfies unanimity, weak independence, weak monotonicity and non-discrimination: for the last, no singleton is located where she self-designates, and so all are treated the same. Clearly there are profiles in which an individual can self-designate in one of the two categories, but be located elsewhere by the majority view of others, and so there are profiles for which accountability does not hold. The majority aggregator also satisfies symmetry.

When $n$ is even and $r=2$, each individual must nominate a tie-breaker. If each individual nominates herself as tie-breaker, unanimity, weak independence, weak monotonicity, non-discrimination and symmetry hold, and again there are profiles for which accountability does not hold. When $r>2$, there are profiles for which there is no majority outcome. A preponderance aggregator locates $i$ in the category that is supported by the largest number of individuals (including $i$ ), but this also needs a tie-breaking mechanism, which now requires that each individual nominates a hierarchy of tie-breakers ${ }^{2}$. For example, if $n=5$ and $r=3$ individual 1 might face 'tied votes' in several ways: if

$$
\Pi_{11}=\Pi_{21}=C_{1}, \Pi_{31}=\Pi_{41}=C_{2}, \Pi_{51}=C_{3}
$$

then $q_{1}=C_{1}$ if individual 1's first-level tie-breaker is herself. If

$$
\Pi_{11}=C_{1}, \Pi_{21}=\Pi_{31}=C_{2}, \Pi_{41}=\Pi_{51}=C_{3}
$$

there is no tie involving individual 1 , and $q_{1}=C_{2}$ if individual 1 's second-level tiebreaker is individual 2. A first-level tie-breaker takes precedence over a second level tie-breaker (and so on if there are more categories and more tie-breakers are needed). This aggregator satisfies unanimity, weak independence, weak monotonicity and non-discrimination: again, for the last, no singleton is located where she selfdesignates, and so all singletons are impacted in the same way.

However, the preponderance aggregator does not satisfy symmetry. As examples, suppose first that every individual nominates herself as first-level tie-breaker, and another individual as second-level tie-breaker. If 1 nominates tie-breakers $1 \& 2$, and 3 nominates tie-breakers $3 \& 4$, the profile

\footnotetext{
${ }^{2}$ The nomination might be made by the person implementing the aggregator, such as the tutor in the grading context.
} 


$$
\Pi=\left[\begin{array}{lllll}
C_{1} & C_{3} & C_{2} & C_{3} & C_{3} \\
C_{2} & C_{3} & C_{2} & C_{3} & C_{3} \\
C_{2} & C_{3} & C_{1} & C_{3} & C_{3} \\
C_{3} & C_{3} & C_{3} & C_{3} & C_{3} \\
C_{3} & C_{3} & C_{3} & C_{3} & C_{3}
\end{array}\right]
$$

yields $q=\left(C_{2}, C_{3}, C_{3}, C_{3}, C_{3}\right)$. This example does not satisfy symmetry applied to $1 \& 3$, but it does satisfy non-discrimination because no singleton can be located where she self-designates.

If every individual nominates $1 \& 2$ as tie-breakers (with 1 taking precedence over 2), the profile

$$
\Pi^{\prime}=\left[\begin{array}{lllll}
C_{1} & C_{3} & C_{3} & C_{3} & C_{2} \\
C_{2} & C_{3} & C_{3} & C_{3} & C_{2} \\
C_{3} & C_{3} & C_{3} & C_{3} & C_{3} \\
C_{1} & C_{3} & C_{3} & C_{3} & C_{1} \\
C_{2} & C_{3} & C_{3} & C_{3} & C_{1}
\end{array}\right]
$$

yields $q^{\prime}=\left(C_{1}, C_{3}, C_{3}, C_{3}, C_{2}\right)$. This example does not satisfy symmetry applied to individuals $1 \& 5$, but it does satisfy non-discrimination because no singleton can be located where she self-designates.

A preponderance aggregator might be regarded as useful in some contexts where accountability is not attractive. It is excluded if symmetry is required, but not if non-discrimination is required.

Sung and Dimitrov (2005) show that the five conditions used by K\&R are not independent, and specifically that the consensus and monotonicity conditions in $\mathrm{K} \& \mathrm{R}$ are entailed by their symmetry and independence conditions and their liberal principle. Their analysis cannot be applied directly to our theorem 1 principally because we have substituted non-discrimination for symmetry. We do not pursue further the question of whether the five conditions of the theorem are independent, but we have established that there are aggregators other than $f^{L I B}$ that satisfy all the conditions except accountability.

\section{Discussion}

K\&R demonstrated an important result in the tradition of Arrow's theorem by showing that only one form of aggregator satisfies a set of arguably attractive conditions, and that disagreement is effectively ignored. In Arrow's context, the aggregators that satisfy the specified conditions (relating to unanimity, independence and monotonicity) involve dictatorship in which all disagreements are ignored because only one person's opinion influences every part of the outcome. In $\mathrm{K} \& \mathrm{R}$, the only aggregator that satisfies the five conditions is $f^{L I B}$ through which disagreement is ignored because each individual determines the outcome as her selfdesignation, regardless of the opinions of others. The results are similar in that both 
lead to the conclusion that compromises between diverse opinions is not possiblebut different in that $f^{L I B}$ gives every individual some influence because, in all circumstances, each determines her own location.

We have adapted the conditions to contexts in which people are allocated to more than two categories. Theorem 1 entails that, if we want to allow that otherdesignations affect some outcome locations, then we must tolerate the violation of one of five conditions. Equivalently, any assertion that the outcome of a census or survey that is based on self-designation does not give an accurate reflection of the numbers in each of several categories must identify which of the five conditions does not hold.

The information base includes only self- and other designations, and so it is difficult to provide any justification for rejecting unanimity. No evidence can be adduced to counter the conclusions that, if every individual (including $i$ ) designates $i$ in $C_{s}$, then the outcome should locate $i$ in $C_{s}$ and that if no individual designates $i$ in $C_{s}$, then the outcome should not locate $i$ in $C_{s}$.

Rejecting weak independence requires the identification of circumstances in which it is reasonable to argue that a change in the outcome location of some individual is the only consequence of re-designations of other individuals, which is arguably implausible in its assumption about the impact of system effects. More general independence conditions entail that the outcome location of $j$ is unaffected by re-designations of other individuals, which denies the possibility of system effects, and are arguably more easily rejected.

Weak monotonicity disallows perverse changes that are not excluded by weak independence and is needed in the proof of theorem 1 only for profile changes where system effects are ruled out through the use of unanimity.

Non-discrimination replaces the symmetry condition used by K\&R and focusses on individuals who are in comparable situations given the profile of self- and other designations. Its application is limited because it applies only to singletons. The condition could be rejected only by asserting some justification of why one $s \backslash t$ singleton should be treated differently from another, despite the similarity of their positions.

The most vulnerable of the conditions is accountability, and it is clear that it might not be acceptable in some contexts-such as the project-grade example. If the condition is not acceptable, there is a profile in which $j$ self-designates in $C_{s}$, but the aggregator leaves $C_{s}$ empty in the outcome for the profile. The accountability narrative is harder to ignore in a straightforward allocation problem (such as of musicians to genres), and someone who wants to reject the results of a census that asks only for designations would need to argue that a category is better left empty than that an individual should be located there just because she self-designates in that category, effectively asserting that there can be circumstances in which no-one is qualified to be in that category despite their self-designation.

In the two-category case, accountability precludes the use of a majority aggregator in which each individual is located where she is designated by a majority of individuals (including herself). This aggregator satisfies the other four conditions: there are no system effects, and all singletons are treated alike in that they are not 
located where they self-designate. Using this aggregator, a category could be empty in the outcome because anyone who self-designates there is 'outvoted' by others. In the multi-category case, a preponderance aggregator satisfies the four other conditions-but not symmetry.

Acknowledgements I am grateful to Eliot Michaelson and anonymous referees for their comments on earlier versions of this paper.

\section{Declarations}

Conflict of interest The author declares that they have no conflict of interest.

Open Access This article is licensed under a Creative Commons Attribution 4.0 International License, which permits use, sharing, adaptation, distribution and reproduction in any medium or format, as long as you give appropriate credit to the original author(s) and the source, provide a link to the Creative Commons licence, and indicate if changes were made. The images or other third party material in this article are included in the article's Creative Commons licence, unless indicated otherwise in a credit line to the material. If material is not included in the article's Creative Commons licence and your intended use is not permitted by statutory regulation or exceeds the permitted use, you will need to obtain permission directly from the copyright holder. To view a copy of this licence, visit http:// creativecommons.org/licenses/by/4.0/.

\section{References}

Alcantud, J. C. R., \& Laruelle, A. (2020). Independent collective identity functions as voting rules. Theory and Decision, 89, 107-119.

Arrow, K. J. (1951). Social choice and individual values: Cowles foundation monograph (Vol. 12). New York: Wiley.

Cho, W. J., \& Ju, B.-G. (2017). Multinary group identification. Theoretical Economics, 12, 513-531.

Fioravanti, F., \& Tohmé, F. (2020). Alternative axioms in group identification problems. Journal of Classification. https://doi.org/10.1007/s00357-020-09378-x.

Galili, L. (2020). The other tribe: Israel's Russian-speaking community and how it is changing the country. Brookings. https://www.brookings.edu/research/the-other-tribe-israels-russian-speakingcommunity-and-how-it-is-changing-the-country/.

Gibbard, A. (1973). Manipulation of voting schemes: A general result. Econometrica, 41, 587-601.

Houy, N. (2007). "I want to be a J!': Liberalism in group identification problems. Mathematical Social Sciences, 54, 59-70.

Kasher, A. (1993). Jewish collective identity. In D. T. Goldberg \& M. Krausz (Eds.), Jewish identity: Temple University Press.

Kasher, A., \& Rubinstein, A. (1997). On the question "Who is a J?', a social choice approach. Logique and Analyse, 160, 385-395.

Miller, A. D. (2008). Group identification. Games and Economic Behavior, 63, 188-202.

Reeves, P. (2011). Russian influx splits Israel over Jewish identity, Independent. https://www. independent.co.uk/news/world/middle-east/russian-influx-splits-israel-over-jewish-identity-699676. html.

Rubinstein, A., \& Fishburn, P. C. (1986). Algebraic aggregation theory. Journal of Economic Theory, 38, 63-77.

Samet, D., \& Schmeidler, D. (2003). Between liberalism and democracy. Journal of Economic Theory, 110, 213-233.

Satterthwaite, M. (1975). Strategy-proofness and Arrow's conditions: Existence and correspondence theorems for voting procedures and social welfare functions. Journal of Economic Theory, 10, 187-217.

Sen, A. K. (1970). The impossibility of a paretian liberal. Journal of Political Economy, 78, 152-157.

Sen, A. K. (2017). Collective choice and social welfare, extended edition. London: Penguin. 
Sung, S.-C., \& Dimitrov, D. (2005). On the axiomatic characterization of "Who is a J?'. Logique et Analyse, 189(192), 101-112.

The Jewish Agency for Israel. (2019). The law of return. http://archive.jewishagency.org/first-steps/ program $/ 5131$.

Wilson, R. (1975). On the theory of aggregation. Journal of Economic Theory, 10, 89-99.

Publisher's Note Springer Nature remains neutral with regard to jurisdictional claims in published maps and institutional affiliations. 